\title{
Vibration of the Duffing oscillator: Effect of fractional damping
}

\author{
Marek Borowiec ${ }^{\mathrm{a}, *}$, Grzegorz Litak ${ }^{\mathrm{a}}$ and Arkadiusz Syta ${ }^{\mathrm{b}}$ \\ ${ }^{a}$ Department of Applied Mechanics, Technical University of Lublin, Nadbystrzycka 36, PL-20-618 Lublin, Poland \\ ${ }^{\mathrm{b}}$ Department of Applied Mathematics, Technical University of Lublin, Nadbystrzycka 36, PL-20-618 Lublin, Poland
}

Received 16 July 2005

Revised 7 January 2006

\begin{abstract}
We have applied the Melnikov criterion to examine a global homoclinic bifurcation and transition to chaos in a case of the Duffing system with nonlinear fractional damping and external excitation. Using perturbation methods we have found a critical forcing amplitude above which the system may behave chaotically. The results have been verified by numerical simulations using standard nonlinear tools as Poincare maps and a Lyapunov exponent. Above the critical Melnikov amplitude $\mu_{c}$, which a sufficient condition of a global homoclinic bifurcation, we have observed the region with a transient chaotic motion.
\end{abstract}

Keywords: Duffing oscillator, Melnikov criterion, chaotic vibration

\section{Introduction}

A nonlinear oscillator with the Duffing term but linear damping is one of the simplest systems leading to chaotic motion studied originally by Ueda [1-3]. The problem of its nonlinear vibrations has attracted researchers from various fields of research; from natural science [4,5] and mathematics [6] to mechanical [8-10] and electrical engineering [1-3]. This system, for a negative linear part of stiffness, shows homoclinic orbits, and the transition to chaotic vibration can be treated analytically by the Melnikov method. Note that such a treatment has already been performed successfully to selected problems with various potentials [6,7]. In spite of fact that a large bibliography is devoted to vibration of a single realization [1-12] or set of coupled Duffing oscillators [13-17] with numerous modifications to potential and forcing parts, the problem of nonlinear damping in chaotically vibrating system has been focused shortly [12]. The fractionally damped Duffing's equation, for a single well potential with a positive linear part and damping defined by a fractional derivative, has been studied in detail by Padovan and Sawicki [18]. They were especially focused on determination of the influence of fractional damping on the frequency amplitude response. Some additional insight into this problem can be also found in the context of self excitation effects [11, $13,16,17,19]$. On the other hand, in the paper of Trueba et al. [12], the systematic discussion on square and cubic damping effects on global homoclinic bifurcations in the Duffing system was given while Awrejcewicz and Holicke applied the Melnikov theory to the Duffing system in a dry friction limit [20]. In the present paper we reexamine the problem of nonlinear damping including fractional damping cases by both; the analytical Melnikov method as well as numerical simulations. Namely we are going to study a single degree of freedom nonlinear oscillator with the Duffing potential and fractional damping:

$$
\ddot{x}+\alpha \dot{x}|\dot{x}|^{p-1}+\delta x+\gamma x^{3}=\mu \cos \omega t,
$$

where $x$ is displacement and $\dot{x}$ velocity, respectively, while the external potential force $F_{x}$ and corresponding potential $V(x)$ (Fig. 1(a)) are defined;

*Corresponding author. E-mails: m.borowiec@pollub.pl,g.litak@pollub.pl. 

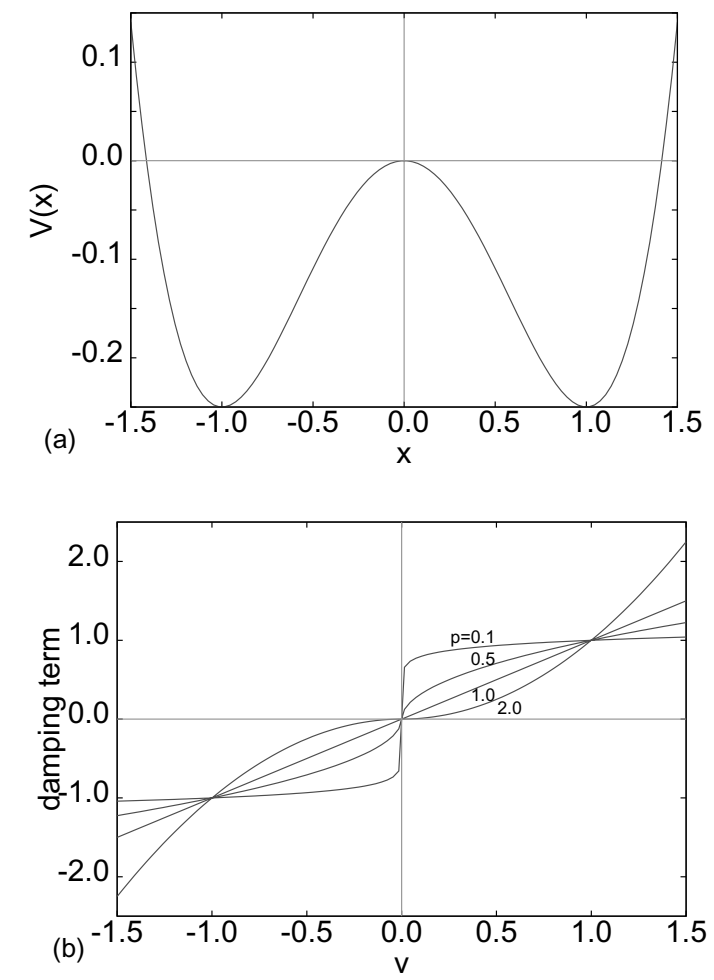

Fig. 1. External potential $V(x)$ for $\delta=-1$ and $\gamma=1$ (Fig. 1(a)); and velocity $(v=\dot{x})$ dependence of a damping term dpt $(\dot{x}) / \alpha$ for different $p$ normalized by $\alpha$ (Fig. 1(b)).

$$
-\delta x-\gamma x^{3}=F_{x}=-\frac{d V}{d x}, \quad V(x)=\frac{\delta x^{2}}{2}+\frac{\gamma x^{4}}{4} .
$$

Here the non-linear damping term is defined by the exponent $p$ :

$$
\operatorname{dpt}(\dot{x})=\alpha \dot{x}|\dot{x}|^{p-1} .
$$

We have plotted the above function versus velocity $(v=\dot{x})$ in Fig. 1(b) for few values of $p$. Note that, the case $p \rightarrow 0$ (see $p=0.1$ in Fig. 1(b) for a relatively small velocity) mimics the dry friction phenomenon [21,22].

\section{Melnikov analysis and numerical simulations}

We start our analysis from the unperturbed Hamiltonian $H^{0}$ :

$$
H^{0}=\frac{v^{2}}{2}+V(x) .
$$

The potential function $V(x)$ (Fig. 1(a)) has the local peak at the saddle point $x=0$. Existence of this point with a horizontal tangent makes homoclinic bifurcations to take place possible. This includes transitions from regular to chaotic solutions. To study effects of damping and excitation, we apply small perturbations around the homoclinic orbits. This implies using a small parameter $\epsilon$ in the Eq. (1) with perturbation terms. Equation (1) can be simultaneously uncoupled into two differential equations of the first order:

$$
\begin{aligned}
& \dot{x}=v \\
& \dot{v}=-\epsilon \tilde{\alpha} v|v|^{p-1}-\delta x-\gamma x^{3}+\epsilon \tilde{\mu} \cos \omega t,
\end{aligned}
$$

where $\epsilon \tilde{\alpha}=\alpha$ and $\epsilon \tilde{\mu}=\mu$, respectively. 


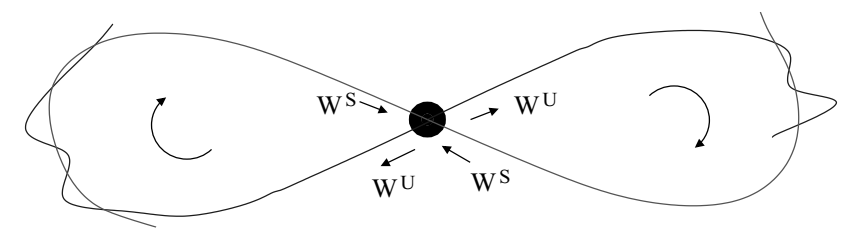

Fig. 2. Perturbed homoclinic orbits Eq. (8): stable and unstable manifolds ( $W^{S}$ and $\left.W^{U}\right)$.
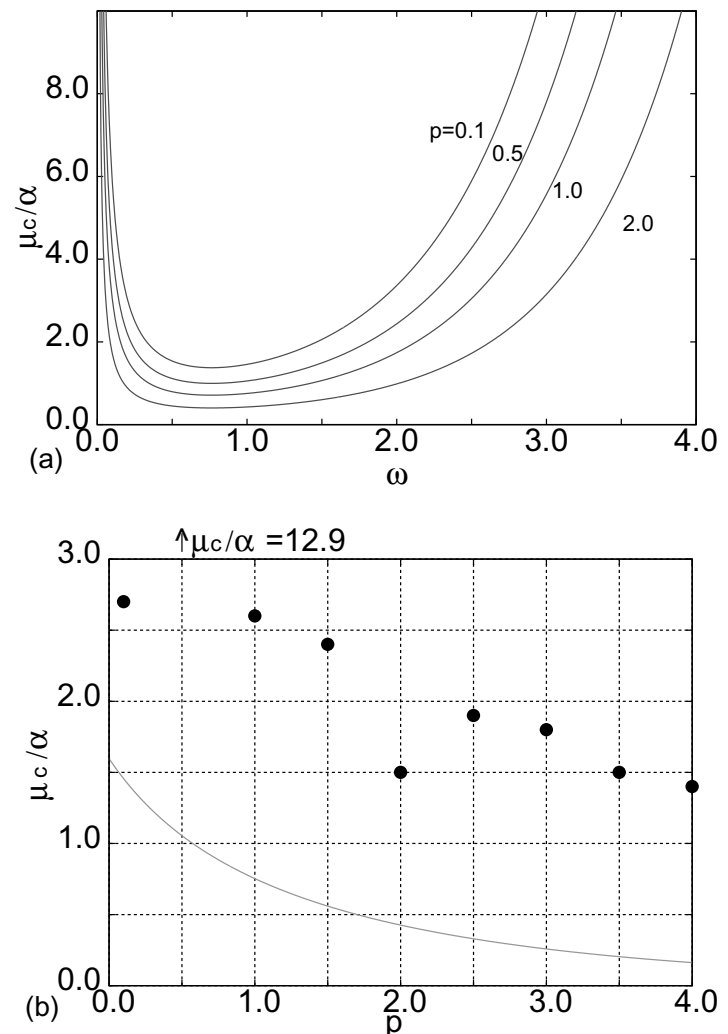

Fig. 3. Critical value of excitation $\mu_{c}$ versus $\omega$ (Fig. 3(a)) and exponent $p$ (Fig. 3(b)) for $\delta=-1, \gamma=1$. Points in Fig. 3(b) show numerical results of transitions to chaos obtained by Lyapunov exponents.

At the saddle point $x=0$, for an unperturbed system (Fig. 1(a)), the system velocity reaches zero, (for infinite time $t= \pm \infty$ ) so the total energy has only its potential part. Transforming Eqs (2) and (4) for a chosen nodal energy $(E=0)$ and for $\delta<0, \gamma>0$ we get the following expression for velocity:

$$
v=\frac{\mathrm{d} x}{\mathrm{~d} t}=\sqrt{2\left(-\frac{\delta x^{2}}{2}-\frac{\gamma x^{4}}{4}\right)} .
$$

Now one can perform integration over $x$ :

$$
t-t_{0}= \pm \int \frac{\mathrm{d} x}{x \sqrt{-\delta-\frac{\gamma x^{2}}{2}}},
$$

where $t_{0}$ represents an integration constant. Finally, we get so called homoclinic orbits (Fig. 2):

$$
x^{*}= \pm \sqrt{\frac{-2 \delta}{\gamma}} \frac{1}{\cosh \left(\sqrt{-\delta}\left(t-t_{0}\right)\right)}
$$



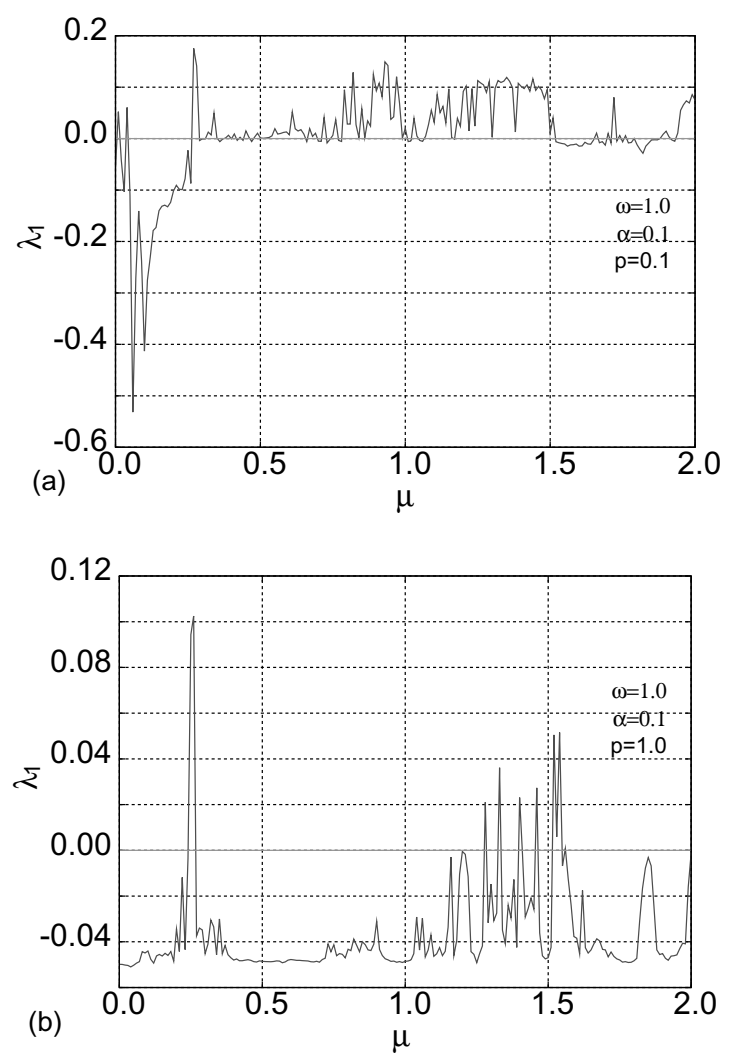

Fig. 4. Lyapunov exponents for $\delta=-1, \gamma=1$ and $p=0.1$ (Fig. 4(a)), $p=1.0$ (Fig. 4(b).

$$
v^{*}= \pm \sqrt{\frac{2}{\gamma}} \delta \frac{\tanh \left(\sqrt{-\delta}\left(t-t_{0}\right)\right)}{\cosh \left(\sqrt{-\delta}\left(t-t_{0}\right)\right)}
$$

where ' + ' and ' - ' signs are related to left - and right - side orbits, respectively (Fig. 2). Note, the central saddle point $x_{0}=0$ is reached in time $t$ corresponding to $+\infty$ and $-\infty$, respectively.

In case of perturbed orbits $W^{S}$ (a stable manifold) and $W^{U}$ (an unstable manifold) the distance between them is given by the Melnikov function $\mathrm{M}\left(t_{0}\right)$ :

$$
M\left(t_{0}\right)=\int_{-\infty}^{+\infty} h\left(x^{*}, v^{*}\right) \wedge g\left(x^{*}, v^{*}\right) d t
$$

where the corresponding differential form $h$ means the gradient of unperturbed Hamiltonian Eq. (4):

$$
h=\left(\delta x^{*}+\gamma\left(x^{*}\right)^{3}\right) \mathrm{d} x+v^{*} d v,
$$

while $g$ is a perturbation form Eq. (5) to the same Hamiltonian:

$$
g=\left(\tilde{\mu} \cos \omega t-\tilde{\alpha} v^{*}\left|v^{*}\right|^{p-1}\right) d x .
$$

All differential forms are defined on homoclinic orbits $(x, v)=\left(x^{*}, v^{*}\right)$ Eq. (9).

Condition for a global homoclinic transition, corresponding to a horse-shoe type of stable and unstable manifolds cross-section (Fig. 2), can be written as:

$$
\bigvee_{t_{0}} \mathrm{M}\left(t_{0}\right)=0 \text { and } \frac{\partial \mathrm{M}\left(t_{0}\right)}{\partial t_{0}} \neq 0
$$



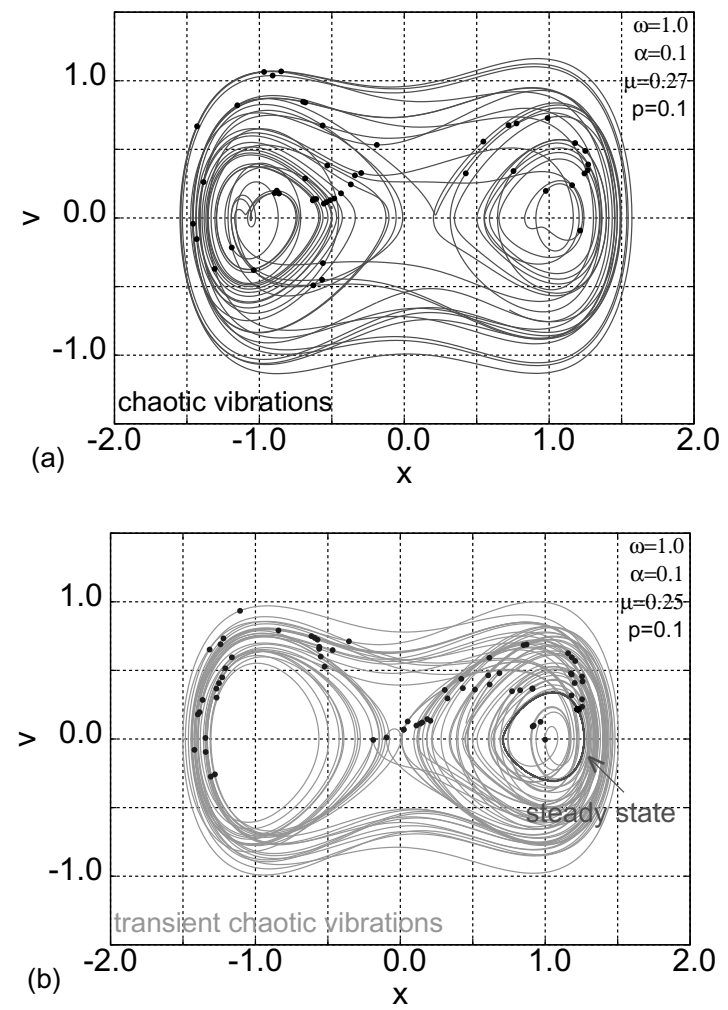

Fig. 5. Phase portraits and Poincare maps for $\delta=-1, \gamma=1$ and $\mu=0.27$ (Fig. 5(a)), $\mu=0.25$ (Fig. 5(b)).

Evaluating the corresponding integral Eq. (9) after some lengthly algebra the last condition Eq. (12) yields a critical value of the excitation amplitude $[4,12] \mu_{c}$ :

$$
\mu_{c}=\alpha \frac{2^{p / 2}}{\pi \omega} \frac{(-\delta)^{p+1 / 2}}{\gamma^{p / 2}} \mathrm{~B}\left(\frac{p+2}{2}, \frac{p+1}{2}\right) \cosh \left(\frac{\pi \omega}{2 \sqrt{-\delta}}\right)
$$

where the $\mathrm{B}(r, s)$ is the Euler Beta function:

$$
B(r, s)=\frac{\Gamma(r) \Gamma(s)}{\Gamma(r+s)}
$$

and $\Gamma(n)$ denotes the Euler Gamma function:

$$
\Gamma(n+1)=n \Gamma(n) .
$$

In Fig. 3(a) we plotted the results of Melnikov analysis for a critical amplitude $\mu_{c} / \alpha$ assuming few values of $p$ while $\delta=-1, \gamma=1$. For $\mu>\mu_{c}$ the system can transit to chaotic vibrations. In the next figure (Fig. 3(b)) we plotted $\mu_{c}$ versus $p$. Here we have also compared the results of analytical investigations represented by a solid line with numerical simulations marked by circle points. The numerical results can give a local criterion of chaotic vibration appearance, namely a positive value of the largest Lyapunov exponent $\lambda_{1}$ [23]. Note that in all cases (Fig. 3(b)) points are above the line. This can be associated with the fact that the Lyapunov exponent is a sufficient criterion of system transition to chaos while the Melnikov condition is only necessary one. Thus we can conclude that both sets of results: analytical and numerical are in good agreement. The examples of the largest Lyapunov exponent $\lambda_{1}$ versus the excitation amplitude $\mu$ are plotted in Fig. 4(a) and (b). In our numerical code we started calculations from the same initial conditions $\left(x_{0}, v_{0}\right)=(0.45,0.1)$ for every new value of $\mu$. One can clearly see the point of $\lambda_{1}$ sign change. This indicates transition to chaotic vibrations. Note that for $p=0.1$ we have got some small but positive values of $\lambda_{1}$ for weak excitation ( $\mu$ close to 0 ). When we examined this case with more details we observed that 

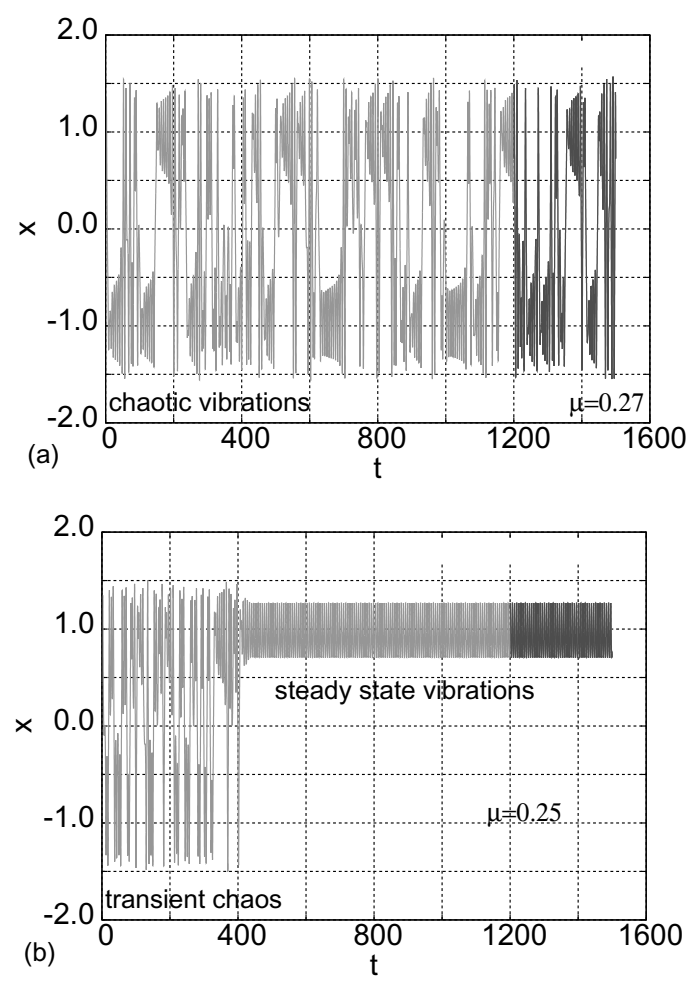

Fig. 6. Time histories for $\delta=-1, \gamma=1$ and $\mu=0.27$ (Fig. 6(a)), $\mu=0.25$ (Fig. 6(b)).

the amplitude of vibrations was very small. Appearance of small positive value of $\lambda_{1}$ seams to be a combined effect of physical phenomenon of dry friction and our assumed numerical integration procedure of constant time steps $\Delta t$ (in most cases we used $\Delta t=2 \pi /(500 \omega)$ ). It is known from the studies of a stick-slip phenomenon [24,25] that in such cases integration should be performed very carefully. Indeed, making time steps relatively smaller (50 times smaller) we checked that it is sufficient to reduce the positive value around $\mu=0$ leaving, in principle, unchanged that $\lambda_{1}$ which appeared at $\mu \approx 0.27$. To reduce this artificial effect of small positive values of $\lambda_{1}$ and assuming an efficient algorithm (not so much time consuming) we decided to put a minimal threshold of $\lambda_{1}=0.1$ above which the system was identified in a chaotic state (see Figs 3(b), 4(a) and 4(b)).

For a given value of $p(p=0.1)$ we show (in Fig. 5(a) for $\mu=0.27$ and Fig. 5(b) for $\mu=0.25)$ typical phase portraits (represented by lines) and simultaneously Poincare maps (represented by points). Note that in Fig. 5(a) we plotted trajectories of chaotic motion for $t \in[1200,1500]$, in units of $1 / \omega$, while for Fig. 5(b) we found transient behaviour. To show differences we plotted corresponding trajectories for $t \in[0,1200]$ and $t \in[1200,1500]$ in various colours. The characteristic time $t=1200$ has been chosen in such a way to reach by our system a steady state. It is easy to see that Fig. 5(a) represents a non-periodic vibration state $(\mu=0.27)$ just after the system transition to a chaotic motion (see function $\lambda_{1}$ versus $\mu$ in Fig. 4(a)). The corresponding Lyapunow exponent $\lambda_{1}=0.175$ is positive indicating on exponential divergence. In spite of a small number of Poincare stroboscopic points we can see a general view of a strange chaotic attractor. On the other hand Fig. 5(b) shows system behaviour state for a smaller excitation amplitude $(\mu=0.25)$. In this case the initial trajectories follow the strange attractor complex geometry but after long enough time $(t=1200)$ collapse to a steady state motion. Here the corresponding Lyapunov exponent $\lambda_{1}=-0.023$ is negative which means a convergent motion.

For better clarity we also plotted the corresponding time histories (Fig. 6(a) and (b)) using the same colour as in Fig. 5(a) and (b). One can easly note that for $\mu=0.27$ (Figs 5(a) and 6(a)) we have chaotic motion of the system while for $\mu=0.25$ (Figs 5(b) and 6(b)) after some time of chaotic transient motion with a large amplitude of oscillations and jumping between potential wells we got steady state regular vibrations of much smaller amplitude located in a single potential well. 


\section{Summary and conclusions}

We have examined criteria for transition to chaotic vibrations in the Duffing system with a damping term $d p t(v)=v|v|^{p-1}$ described by a term fractional exponent $p$. We have been interested especially in $p<1.0(p=0.1)$ representing an important case which can be associated with a dry friction phenomenon [22]. The critical value of the excitation amplitude $\mu$ above which the system vibrate chaotically has been estimated, in the first step, by means of the Melnikov method and later confirmed by calculating corresponding Lyapunov exponent.

The Melnikov method is sensitive to a global homoclinic bifurcation and gives a necessary condition for excitation amplitude $\mu=\mu_{c 1}$ system in its transition to chaos [6,7]. On the other hand the largest Lyapunov exponent [23], measuring the local exponential divergences of particular phase portrait trajectories gives a sufficient condition $\mu=\mu_{c 2}$ for this transition which has obviously a higher value of the excitation amplitude $\mu=\mu_{c 2}>\mu_{c 1}$.

Above the Melnikov transition predictions $\left(\mu>\mu_{c 1}\right)$ we have got transient chaotic vibrations [7-9] as we expected drifting to a regular steady state away the fractal attraction regions separation boundary. This is typical behaviour of the system which undergoes global homoclinic bifurcation. In the region of resonance the amplitude of vibration depends on the damping exponent in a nontrivial way. In the particular case [18] amplitude response is rising for small fractional exponents $(p<1)$. Namely, such increase can be identified as an analogues of increasing system response on stronger external forcing. Thus one may expect that a chaotic threshold would be reached easier in that case. In fact, comparing Fig. 4(a) and (b), we observe larger Lyapunov exponents for fractional $p$ ( $p=0.1$ in Fig. 4(a). However from our Melnikov theory analysis we draw a different conclusion. The cases for $p<1$ (Fig. 3(a-b)) seem to be more stable against transition to chaos then the $p=1$ case. This interesting contradiction should be clarified by further simultaneous studies of local bifurcations and resonances in the above system.

For small $p$ and in the limit of a small velocity we have found a region of system parameters leading to an interesting dry friction effect. Note, in our approach this limit has been obtained naturally on same footing as other realizations. This enabled us to compare results of various damping terms (Figs 1(b) and 3(b)). However to get reliable results in that region one must improve a simple numerical algorithm. We have not studied this effect systematically in this paper leaving it for a future publication.

The present approach can be used to generalize models of magnetorheological dampers in novel studies of their influence on vehicle dynamics [19]. To reduce harmful vibrations one can consider an application of dampers composed of fractional terms.

\section{Acknowledgments}

This paper has been partially supported by the Polish Ministry of Science and Informatization. Authors would like to thank organizers of the Conference Recent Advances in Nonlinear Mechanics in Aberdeen (2005) for giving them an opportunity to preset these results. GL would like to thank the Max Planck Institute for the Physics of Complex Systems for hospitality.

\section{References}

[1] Y. Ueda, Randomly transitional phenomena in the system governed by Duffing's equation, J. Stat. Phys. 20 (1979), 181-196.

[2] Y. Ueda, Steady motions exhibited by Duffing's equations: a picture book of regular and chaotic motions, in: New Approaches to Nonlinear Problems in Dynamics, P.J. Holmes, ed., SIAM, Philadelphia 1980, pp. 331-322.

[3] Y. Ueda and N. Akamatsu, Chaotically transitional phenomena in the forced negative-resistance oscillator, IEEE Transactions on Circuits and Systems 28 (1981), 217-224.

[4] F.C. Moon and P.J. Holmes, A magetoelastic strange attractor, Journal of Sound and Vibration 65 (1979), $275-296$.

[5] M. Zalalutdinov, K.L. Aubin, M. Pandey, A.T. Zehnder, R.H. Rand, H.G. Craighead, J.M. Parpia and B.H. Houston, Frequency entrainment for micromechanical oscillator, Applied Phys. Lett. 83 (2003), 3281-3283.

[6] J. Guckenheimer and P. Holmes, Nonlinear Oscillations, Dynamical Systems and Bifurcations of Vectorfields, Springer, New York 1983.

[7] S. Wiggins, Introduction to Applied Nonlinear Dynamical Systems and Chaos, Spinger, New York 1990.

[8] W. Szemplińska-Stupnicka and J. Rudowski, Bifurcations phenomena in a nonlinear oscillator: approximate analytical studies versus computer simulation results, Physica D 66 (1993), 368-380. 
[9] W. Szemplińska-Stupnicka, The analytical predictive criteria for chaos and escape in nonlinear oscillators: A survey, Nonlinear Dynamics 7 (1995), 129-147.

[10] F.C. Moon, Chaotic Vibrations, an Introduction for Applied Scientists and Engineers, John Wiley \& Sons, New York 1987.

[11] G. Litak, G. Spuz-Szpos, K. Szabelski and J. Warmiński, Vibration analysis of self-excited system with parametric forcing and nonlinear stiffness, Int. J. Bifurcation and Chaos 9 (1999), 493-504.

[12] J.L. Trueba, J. Rams and M.A.F. Sanjuan, Analytical estimates of the effect of nonlinear damping in some nonlinear oscillators, Int. J. Bifurcation and Chaos 10 (2000), 2257-2267.

[13] J. Warmiński, G. Litak and K. Szabelski, Vibrations of a parametrically and self-excited system with two degrees of freedom in identification in engineering systems, in: Proc. of Second International Conference on Identification in Engineering Systems, M.I. Friswell, J.M. Mottershead and A.W. Lees, eds, Swansea, March 1999, University of Wales Swansea 1999), pp. 285-294.

[14] J. Warmiński, G. Litak and K. Szabelski, Synchronisation and chaos in a parametrically and self-excited system with two degrees of freedom, Nonlinear Dynamics 22 (2000), 135-153.

[15] R. Lifshitz and M.C. Cross, Response of parametrically driven nonlinear coupled oscillators with application to micromechanical and nanomechanical resonator arrays, Phys. Rev. B 67 (2003), 134302

[16] A. Maccari, Parametric excitation for two internally resonant van der Pol oscillators, Nonlinear Dynamics 27 (2002), 367-383.

[17] A. Maccari, Multiple external excitations for two non-linearly coupled Van der Pol oscillators, Journal of Sound and Vibration 259 (2003), 967-976.

[18] J. Padovan and J.T. Sawicki, Nonlinear Vibration of Fractionally Damped Systems, Nonlinear Dynamics 16 (1998), $321-336$.

[19] S. Li, S. Yang and W. Guo, Investigation on chaotic motion in histeretic non-linear suspension system with multi-frequency excitations, Mechanics Research Communications 31 (2004), 229-236.

[20] J. Awrejcewicz and M.M. Holicke, Melnikov's method and stick-slip chaotic oscillations in very weakly forced mechanical systems, Int. J. Bifurcation and Chaos 9 (1999), 505-518.

[21] C.A. Brockley and P.L. Ko, Quasi-harmonic friction - induced vibration, ASME Journal of Lubrication Technology 92 (1970), $550-556$.

[22] R.A. Ibrahim, Friction - induced vibration, chatter, squeal, and chaos. Part I: Mechanics of contact and friction, Appl. Mech. Rev. 47 (1994), 209-226.

[23] A. Wolf, J.B. Swift, H.L. Swinney and J.A. Vastano, Determining Lyapunov exponents from a time-series, Physica D 16 (1985), $285-317$.

[24] U. Galvanetto and S.R. Bishop, Dynamics of a simple damped oscillator undergoing stick - slip vibrations, Meccanica 34 (1999), $337-347$.

[25] R.I. Leine, D.H. Van Campen and B.L. Van de Vrande, Bifurcations in nonlinear discontinuous systems, Nonlinear Dynamics 23 (2000), $105-164$. 

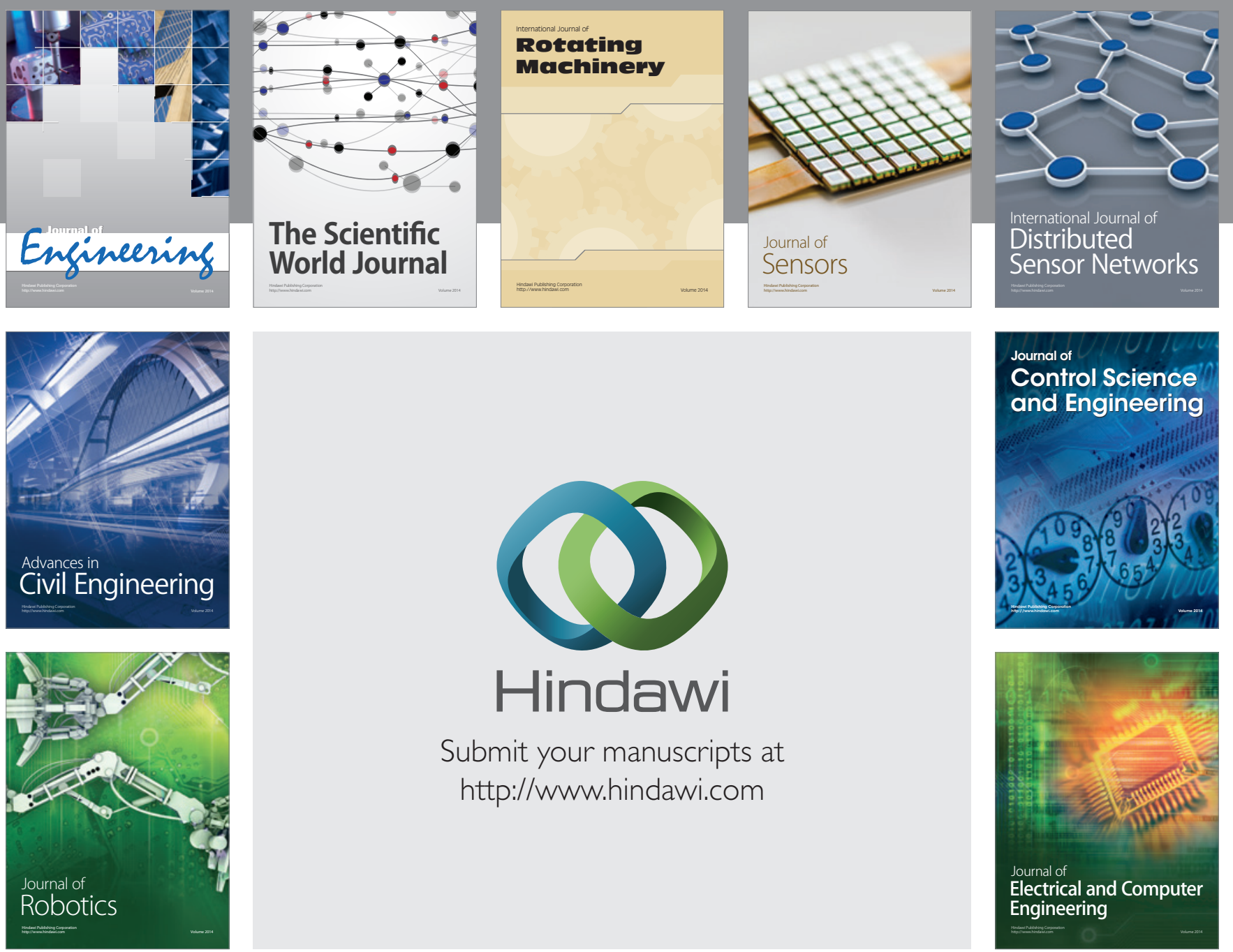

Submit your manuscripts at

http://www.hindawi.com
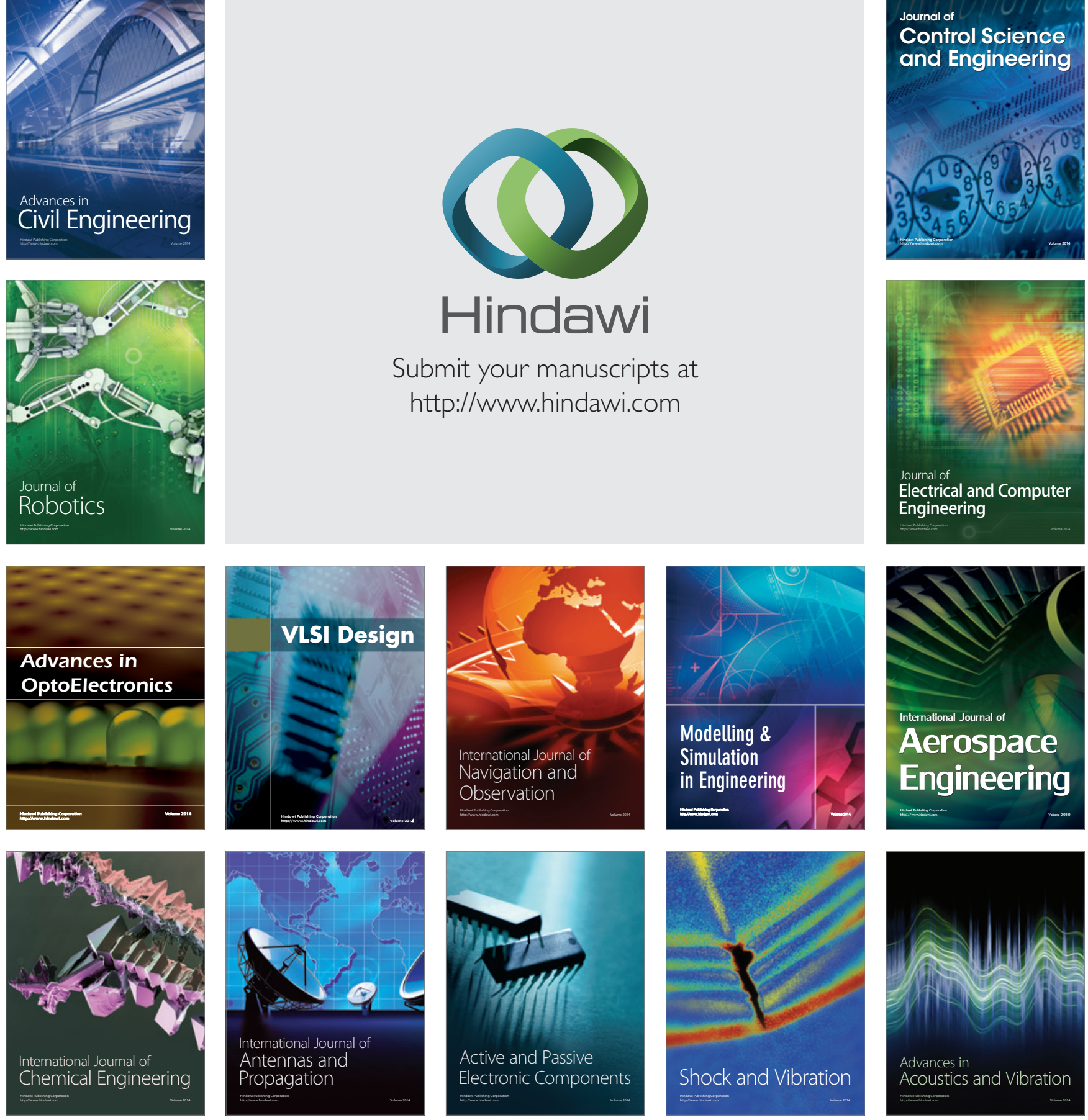